\title{
Essais
}

ESSAIS

Revue interdisciplinaire d'Humanités

$15 \mid 2019$

Jouer l'histoire

\section{Du salon à la salle de classe : Apprendre la Première Guerre mondiale avec Battlefield 1}

William Brou

\section{OpenEdition}

1 Journals

Édition électronique

URL : http://journals.openedition.org/essais/1538

DOI : $10.4000 /$ essais. 1538

ISSN : 2276-0970

Éditeur

École doctorale Montaigne Humanités

Édition imprimée

Date de publication : 15 octobre 2019

Pagination : 77-90

ISBN : 979-10-97024-07-9

ISSN : 2417-4211

Référence électronique

William Brou, «Du salon à la salle de classe : Apprendre la Première Guerre mondiale avec Battlefield 7

», Essais [En ligne], 15 | 2019, mis en ligne le 05 octobre 2020, consulté le 17 octobre 2020. URL:

http://journals.openedition.org/essais/1538; DOI : https://doi.org/10.4000/essais.1538 


\section{Du salon à la salle de classe : apprendre la Première Guerre mondiale avec Battlefield $1^{1}$}

William Brou

La Première Guerre mondiale est un sujet important dans nos programmes scolaires d'Histoire ${ }^{2}$. Ces derniers nous invitent à mettre l'accent sur " les violences extrêmes " subies par les combattants et les civils, mais aussi «l'expérience combattante dans une guerre totale » en classe de Première générale. Le programme de Première technologique est d'autant plus précis qu'il parle de « la violence de guerre dans ses diverses formes et sa répercussion sur les sociétés ". Au-delà des enjeux disciplinaires relatifs à l'apprentissage de l'Histoire, le ministère nous invite à " [former] les élèves à maîtriser ces outils numériques et préparer le futur citoyen à vivre dans une société dont l'environnement technologique évolue constamment ". Par leurs savoirs, les enseignants doivent :

Évoquer les enjeux de la maîtrise du numérique et des technologies [afin qu’ils soient] perçus et compris par les élèves et futurs citoyens. Il est indispensable de les accompagner vers une véritable maîtrise des concepts leur permettant d'être des utilisateurs avisés des outils, services et ressources dans une société de l'information et de la communication en rapide évolution. ${ }^{3}$

Parallèlement à cet engagement, le ministère a inscrit en 2015, par la mise en place d'un nouveau socle de compétences, l'apprentissage de plusieurs langages, dont celui de l'informatique (codage), des médias et des arts, les élèves devant comprendre les différents modes de production et le rôle de l'image :

1 Cet article reprend dans une forme plus développée la proposition faite sur le site des Clionautes et présentée sur ma chaîne YouTube Histoire en Jeux : www.youtube.com/c/HistoireenJeux.

2 Voir la rubrique "Contenus et pratiques d'enseignement " sur le site éduscol spécialisé dans l'information et l'accompagnement des professionnels de l'éducation : http://eduscol.education.fr/pid33035/contenus-et-pratiques-d-enseignement.html (dernière visite le 28/11/2018).

3 "L'utilisation du numérique à l'école ", education.gouv.fr, mis à jour en février 2017 : http:// www.education.gouv.fr/cid208/l-utilisation-du-numerique-a-l-ecole.html (dernière visite le 28/11/2018). 
Lélève apprend à utiliser avec discernement les outils numériques de communication et d'information qu'il côtoie au quotidien, en respectant les règles sociales de leur usage et toutes leurs potentialités pour apprendre et travailler. Il accède à un usage sûr, légal et éthique pour produire, recevoir et diffuser de l'information. Il développe une culture numérique. ${ }^{4}$

Il s'agit ici d'une invitation à utiliser les jeux dans l'enseignement de l'Histoire-Géographie, le cas de l'Histoire étant bien plus problématique en ce que ces productions vidéoludiques peuvent concurrencer le discours historien. Les joueurs vont-ils croire un jeu leur permettant de "vivre la guerre », ou bien l'historien-professeur qui raconte et montre la guerre à travers des documents sources?

Battlefield 1 (BF1) est un jeu de tir à la première personne (FPS) développé par le studio suédois DICE et édité par le géant américain Electronics Arts. C'est le cinquième épisode principal de la série vidéoludique qui a débuté en 2003 avec Battlefield 1942, qui prenait place durant la Seconde Guerre mondiale. À l'instar des autres Battlefield, le jeu possède une campagne solo et une section multijoueurs qui, d'ailleurs, attire la majorité des acheteurs. La campagne est composée de six " récits de guerre " qui placent les joueurs dans la peau de plusieurs soldats répartis sur plusieurs fronts. Dans $B F 1$, il n'existe pas de récit chronologique de la guerre : chaque scénario est introduit par une cinématique de contextualisation, tandis que les missions se jouent à la première personne. Le joueur n'en voit donc que les mains et les armes qu'il manipule, la souris, ou la manette, devenant ainsi de véritables prolongements des bras du soldat. Par le truchement de l'interactivité, le jeu fait ici vivre au joueur une expérience de guerre alors que celui-ci est tranquillement installé dans son salon.

"Battlefield 1 n'est pas un jeu sur la Première Guerre mondiale. " C'est ainsi que Stéphanie Trouillard titrait sa critique du jeu vidéo d'EA, nouveau titre de la série des studios DICE, sortis le 21 octobre 2016. Pour certains, il serait donc inutile de chercher une vérité historique dans ce jeu. D'ailleurs, les joueurs ne semblent pas s'y intéresser, comme si finalement ces derniers achetaient n'importe quel jeu de guerre sans se soucier du contexte utilisé. Sébastien Genvo réaffirme cette réalité dans sa définition des jeux vidéo, en ce qu'ils sont des "systèmes informatiques dont les interfaces d'entrées et de sorties substituent chez l'utilisateur des stimuli artificiels aux stimuli de l'environnement réel immédiat. ${ }^{6}$ Cela suppose donc un travail de la part

4 "Socle commun de connaissances, de compétences et de culture ", Bulletin officiel, $\mathrm{n}^{\circ} 17$, 23 avril 2015, sur le site education.gouv.fr: http://www.education.gouv.fr/pid25535/bulletin officiel.html?cid_bo=87834 (dernière visite le 28/11/2018).

5 Stéphanie Trouillard, "Battlefield 1 n'est pas un jeu sur la Première Guerre mondiale », France 24. com, 5 novembre 2016 : https://www.france24.com/fr/20161105-battlefield-1-jeu-video-premiere-guerre-mondiale-critique-historien-test-gameplay (dernière visite le 28/11/2018)

6 Sébastien Genvo, Introduction aux enjeux artistiques et culturels des jeux vidéo, Paris, L’Harmattan, 2002 , p. 30. 
d'un certain nombre de personnes, que nous appellerons maladroitement les développeurs (game designer, level designer, sound designer), visant à produire des émotions, ces fameux "stimuli ». En effet, si mes jeunes élèves de Troisième n'ont théoriquement pas le droit de jouer à ce jeu (PEGI 18), force est de constater que nombre d'entre eux le connaissaient. Ainsi, il est de notre devoir d'enseignant et d'historien de prendre en main cet objet et d'apprendre à nos élèves à l'analyser comme n'importe quel autre document historique. L'utilisation de $B F 1$ en classe permet-elle de construire des savoirs scientifiques sur la Première Guerre mondiale dans un milieu scolaire ? Je me propose d'exposer ici le dispositif utilisé avec mes élèves de Troisième, d'analyser certaines critiques du jeu réalisées par ces derniers avant d'explorer les enjeux culturels relatifs au jeu d'EA.

\section{Utiliser Battlefield 1 en classe : le dispositif}

Le jeu Battlefield 1 prend place dans un cours somme toute assez classique sur la Première Guerre mondiale. Introduite par une présentation spatiotemporelle du conflit, une première partie développe l'expérience combattante durant le premier conflit mondial (I). C'est dans ce temps que j'utilise le jeu. Je poursuis avec une étude sur l'expérience des civils pendant la guerre (II). Je conclus ensuite mon chapitre avec une étude sur les bilans humains et matériels ainsi que sur les transformations territoriales de l'Europe (III). La séquence proposée ici introduit la première partie du cours et est réalisable en deux ou trois heures; elle s'organise en trois temps.

\section{Temps 1 : Quelle expérience de guerre, le jeu me transmet-il ?}

Dans un premier temps, les élèves prennent connaissance du jeu en en visionnant plusieurs extraits. Deux solutions sont envisageables : la première consiste à diffuser un montage aux élèves ${ }^{7}$, tandis que la seconde diffuse le jeu en direct à l'aide d'une console de jeu, le professeur gardant le contrôle de la manette. Cette seconde solution permet une meilleure interactivité et de meilleures analyses du level design et du game design avec les élèves (voir temps 3). Toutefois, cela suppose d'avoir le matériel et de savoir manipuler le jeu. Dans les deux cas, les jeunes ne manipulent jamais le jeu et demeurent des observateurs.

Dans ce premier temps, il est impossible de visionner tous les « récits de guerre ", d'autant plus que chaque récit est composé de plusieurs missions. Mon choix s'est porté sur trois d'entre eux : le premier récit de guerre introductif

7 Montage disponible à cette adresse : https://youtu.be/xzCz6XS71SA (dernière visite le 28/11/2018). 
est remarquable ; tutoriel du jeu, cette séquence de quinze minutes réunit en un seul lieu de nombreuses informations sur la Première Guerre mondiale. Le joueur interprète successivement six soldats qui manipulent des armes différentes et qui sont confrontés à des difficultés différentes. L'un d'eux monte dans un char britannique Mark II, tandis que l'on peut apercevoir l'aviation de combat et de nouvelles armes telles que les gaz de combat. Le deuxième récit, "Dans la Boue et le Sang ", permet d'aborder le développement de l'artillerie de guerre. Le joueur interprète ici le conducteur d'un char d'assaut Mark II lors de la deuxième bataille de Cambrai. La mission " Des amis haut placés » nous met quant à elle dans la peau d'un aviateur; elle permet d'aborder le rôle de l'aviation de reconnaissance et l'existence d'un No man's land.

Durant cette étape, les élèves sélectionnent des informations que le jeu leur transmet. Ils sont invités à noter ce qu'ils voient, ce qu'ils entendent, ce qu'ils lisent et ce qu'ils ressentent. Il ne s'agit pas de décrire l'intégralité des missions ; on peut guider leur regard à l'aide d'un questionnaire. Dans le cas d'une analyse en direct, les élèves discutaient entre eux avant d'indiquer des informations sur leur feuille. L'enseignant devait quant à lui s'empêcher d'y répondre avant la fin de la séquence.

Temps 2 : Quelle a été l'expérience de guerre d'après les documents sources?

Dans un second temps, les élèves se confrontent à un corpus documentaire. À l'instar d'un historien, ils doivent vérifier les informations. Ici, les documents sources sont répartis en huit dossiers ${ }^{8}$ :

- Présence de soldats noirs dans les armées

- Trêves et fraternisations sur le front

- Les tranchées : lieu de combat et lieu de vie

- Les assauts : des événements meurtriers et dramatiques

- Les chars d'assaut : de nouvelles armes destructrices

- Le No man's land: un espace désertique?

- Les blessés et les morts

- L'aviation

Les dossiers ont été construits pour être en adéquation avec les attentes du programme de Troisième en termes de connaissances et cohérent avec les informations fournies par le jeu. Le corpus documentaire est diversifié ; on y trouve des photos d'archives, des lettres de poilus, des tableaux statistiques, des pièces de musée. L'un des objectifs est de faire travailler les élèves sur des documents variés. Le temps n'étant pas extensible, il n’y a pas de travail

8 Le corpus documentaire est disponible sur le site des Clionautes: https://www.clionautes.org/ categorie/jeux-video-et-histoire (dernière visite le 28/11/2018). 
critique sur les documents sources même s'il serait très intéressant de le faire avec les élèves. Afin de fournir une comparaison plus fine, il est possible de se concentrer sur un cadre spatio-temporel précis, comme la bataille de Verdun ou le Chemin des Dames. Si ce temps est somme toute plus classique dans son approche, il permet d'introduire une nouvelle question : Que m'apprennent ces documents sur l'expérience de guerre des soldats?

\section{Temps 3 : Comparaison}

Dans ce troisième temps, les élèves comparent les informations transmises dans le jeu avec celles relatives aux documents d'archives. L'objectif n'est pas de réaliser un fact-checking complet en ce qu'ils doivent au contraire créer des liens entre les informations. En passant d'un média à un autre, les élèves s'aperçoivent en effet que les images du jeu qu'ils ont sous les yeux sont des constructions créées à partir de ces mêmes documents d'archives, ce qui leur permet de créer du sens entre les documents. Ce temps de comparaison est aussi un temps de discussion. C'est un moment privilégié pour évoquer et peut-être définir avec eux ce qu'est le level design, le game design et le sound design. Autrement dit, il s'agit de comprendre comment un jeu vidéo est créé et réfléchi par les développeurs tout en s'interrogeant sur sa capacité à transmettre des informations par les images, les sons et le texte.

Au-delà de la critique du jeu dont ils ne sont pas experts, les élèves ont manipulé des documents sources qu'ils ont utilisés pour la critique d'un autre document. Bien que difficile pour eux, ce travail d'argumentation et de croisement des sources est fondamental, car il permet d'aborder la question du statut de la source. Qu'est-ce qu'un document source? Est-ce que toutes les images se valent? Dois-je croire tout ce que je vois? Dans le cadre d' une utilisation du jeu en classe de Première, il est également possible de glisser des documents pièges dans les corpus de documents. Il est possible d'insérer un extrait d'un film ou d'un autre jeu vidéo sur la Première Guerre mondiale, comme Verdun'. $S$ 'il est difficile de rendre compte de la totalité des comparaisons, tant elles sont nombreuses, on peut toutefois dégager trois grands axes.

\section{Apprendre avec et sur le jeu : un travail critique}

Je reprends ici la grille d'analyse développée par Yvan Hochet ${ }^{10}$. Pour lui, il y a trois niveaux d'apprentissage avec le jeu vidéo :

9 Jeu sorti en 2015, développé et édité par M2H et Blackmill Games.

10 Yvan Hochet, "Jeu vidéo et enseignement de l'Histoire et de la Géographie ", Les Jeux vidéo comme objet de recherche, Paris, Questions théoriques, 2012, p. 123-134. 
- le joueur peut apprendre avec le jeu, le jeu étant un stimulus,

- le joueur peut apprendre sur le jeu, ce dernier étant le support d'un travail critique,

- le joueur peut apprendre autour du jeu, le jeu étant remplacé dans son contexte.

Dans cette partie, je développerais quelques exemples de comparaisons possibles entre le jeu et les documents du corpus, soit un apprentissage avec et sur le jeu. Tous les exemples proviennent de la première mission du jeu.

\section{Critiquer les images : l'exemple du champ de bataille}

La vue est le premier sens à être mobilisé par les joueurs. Dans $B F 1$, les images peuvent être celles d'une cinématique ou les éléments visuels vus lors des phases de jeu, les premières étant du ressort du scénariste tandis que les secondes sont conditionnées par les level designers ${ }^{11}$. La première mission nous permet d'arpenter un véritable champ de ruines. Il est intéressant de faire noter aux élèves les détails visuels : les maisons détruites, les arbres en feu, les trous d'obus. Des détails visuels qui montrent la violence des combats, le rôle de l'artillerie, et qui sont finalement présents pour susciter l'émotion du joueur. Les images d'archives présentes dans le corpus documentaire permettent quant à elles de montrer la réalité des destructions lors de la Première Guerre mondiale. Les nombreuses photos aériennes qui ont été prises après la guerre sont là pour témoigner de la violence des bombardements d'artillerie. On peut aussi citer les travaux d'Emmanuelle Danchin qui a bien montré comment les ruines ont été instrumentalisées par les pouvoirs en place afin de dénoncer la violence des combats ${ }^{12}$. Il y a d'ailleurs de nombreuses expériences pédagogiques qui utilisent l'étude des paysages pour analyser la violence de la guerre.

Plus loin, dans la même mission du jeu, le joueur doit monter dans un char d'assaut. L'instant est très bref, mais dans notre viseur, nous apercevons deux brancardiers qui transportent un blessé non loin d'une église en ruine. Peu de personnes interrogent la raison de la présence de ces soldats. Bien qu'ils n’apportent rien au scénario en ce qu'ils ne sont qu'un élément de décor, ils soutiennent toutefois l'histoire et le récit du narrateur. Cela signifie qu'il était important de les placer à cet endroit, leur implémentation répondant à un choix des développeurs. Finalement, en lisant une lettre de Charles Guinant, nous découvrons une troublante ressemblance avec la scène visible dans le jeu : "Plus tard, les médecins et infirmiers vinrent me chercher pour m’emmener

11 Le level designer est chargé de construire les différents niveaux (ou cartes) d'un jeu vidéo à travers lesquels le joueur sera amené à évoluer. Il travaille en équipe sous la responsabilité du game designer.

12 Emmanuelle Danchin, Le Temps des ruines, 1914-1921, Rennes, Presses Universitaires de Rennes, 2015. 
à l'hôpital, aménagé dans une ancienne église bombardée. ${ }^{13}$ Si dans un cas comme dans l'autre le visuel vient appuyer le discours sur la violence des combats, l'on voit bien comment les level designers sont allés chercher l'inspiration dans des visuels disponibles dans les archives.

\section{Un gameplay à critiquer : le héros invincible}

Dans la première mission du jeu, toujours, l'on peut demander aux élèves de se concentrer sur le gameplay, étant entendu que le gameplay correspond à « l'ergonomie du jeu, la façon dont on y joue, la facilité avec laquelle on accomplit certaines actions $»^{14}$. Les élèves s'intéresseront aux actions que peut faire le joueur. Durant cette mission, celui-ci est amené à apprendre les bases du gameplay, les déplacements, la visée, les changements d'armes et l'utilisation du masque à gaz, toutes ces informations étant soigneusement indiquées sur l'interface (HUD) qui permet une lecture claire et explicite du jeu. Si notre efficacité au combat est rendue plus facile grâce à cette interface, il ne vient pas naturellement à l'idée des élèves de relever son existence tant elle est " normale " dans un jeu vidéo. Or, aucun document d'archive ne mentionne de telles interfaces. Les comparaisons avec des super-armures comme celles d'Iron Man facilitent ainsi la prise de conscience de cette mise en scène extravagante. Il faut le dire, les soldats de la Première Guerre mondiale étaient jeunes, inexpérimentés pour la plupart et ne savaient pas bien tirer.

En revanche, les élèves ont tout de suite été surpris par la capacité du personnage à régénérer sa santé sans aucune assistance médicale : un contresens historique! Aurait-on imaginé une telle chose à l'époque ? De plus, tandis que la santé de notre soldat diminue, le temps se ralenti, créant une sorte de bullet time afin d'améliorer l'analyse de la situation et ainsi mieux viser et mieux survivre. Ajoutons enfin qu'il est impossible de blesser ses alliés, que ce soit avec des armes légères ou des armes lourdes. Ces éléments de gameplay sont bien évidemment présents pour faciliter le jeu, le rendre plus agréable et surtout moins punitif : ce n'est pas une simulation de guerre. En fin de compte, peu importe votre efficacité au combat, la mission ne peut avancer qu'avec la mort du soldat. La mort devient ici un mal nécessaire pour avancer.

13 Lettre de Charles Guinant, brigadier, 58 é régiment, le 18 octobre 1916, Verdun. Disponible sur le site defense.gouv.fr : https://www.defense.gouv.fr/english/actualites/articles/verdun2016-la-logistique-au-coeur-de-la-bataille-3-3 (dernière visite le 06/12/2018). Voir également, Jean-Pierre Guéno et Yves Laplume (éds.), Paroles de Poilus : Lettres de la Grande Guerre, Paris, Tallandier, 2003.

14 Définition du gameplay sur le site gameart.eu : www.gameart.eu/publi/dossiers/lexique/ gameplay.html, (dernière visite le 06/12/2018). 


\section{Un scénario à critiquer : l'exemple de l'assaut}

Le processus narratif de la mort peut dès lors paraitre choquant. En effet, durant la première mission nous n'incarnons pas un seul soldat, mais six. Et nous devons mourir pour que la mission se poursuive. À chaque mort, le nom et les dates de vie du soldat tombé apparaissent ; la caméra se déplace vers l'arrière pour prendre place dans un nouveau soldat. Pendant ce temps-là, le narrateur prend la parole. Ce processus narratif peut paraitre horrible, mais il explicite la dureté de la guerre et la violence subie par les soldats. Le mouvement de caméra vient appuyer une réalité : si la première ligne tombe, l'arrière doit venir en renfort. Les dates permettent alors de mettre un âge sur ces soldats que l'on ne voit pas. En comparant avec des dossiers militaires disponibles sur le site du Grand mémorial ${ }^{15}$, les élèves se rendent ainsi compte de la jeunesse des soldats.

Cet assaut nous montre également une avalanche d'armes et surtout une coprésence de toutes les composantes de l'armée. Or, à aucun moment de la Grande Guerre n'a été vue une telle co-intervention del'infanterie, de l'artillerie d'assaut et l'aviation. Cette séquence de quinze minutes est définitivement paroxystique tant elle synthétise en un temps très court tout ce que le soldat aurait pu vivre. Un parallèle peut être fait avec le scénario du film Joyeux Noël de Christophe Barratier. Sans oublier que les deux œuvres n'ont pas le même objectif, force est de constater que la trêve présentée dans le film est un condensé d'expériences qui se sont déroulées sur la totalité du front et non sur une seule section. Cette trêve vient clore l'assaut de cette première mission, la pluie laissant sa place au soleil. Les deux soldats survivants baissent leurs armes alors que le narrateur reprend la parole pour introduire la cinématique générique du jeu.

\section{Les enjeux culturels : apprendre autour du jeu}

Travailler sur BF1 permet aussi de questionner la société et l'époque relatives à sa conception. C'est le cour du travail de l'historien que de replacer une production, fût-elle numérique, dans son contexte de production. Il se trouve que $B F 1$ n'a pas été développé par hasard.

\section{Un objet culturel sur la Première Guerre mondiale}

Pour interroger correctement le jeu d'EA, il faut faire intervenir la notion d'objet culturel. Pour étudier une production culturelle, l'historien doit chercher, selon Carle Schorske, "à situer et à interpréter l'œuvre dans le temps et à l'inscrire à la croisée de deux lignes de forces " :

15 Voir la rubrique "Grand Mémorial " sur le site du ministère de la Culture : http://www. culture.fr/Genealogie/Grand-Memorial (dernière visite le 06/12/2018). 
l'une verticale, diachronique, par laquelle il relie un texte ou un système de pensée à tout ce qui les a précédés dans une même branche d'activité culturelle, l'autre horizontale, synchronique, par laquelle l'historien établit une relation entre le contenu de l'objet intellectuel et ce qui se fait dans d'autres domaines à la même époque. ${ }^{16}$

$S^{\prime}$ il est nécessaire de regarder le contenu de $B F 1$, il faut également replacer ce jeu dans une histoire des jeux vidéo sur la Première Guerre mondiale, et en même temps dans son contexte de création. Les jeux vidéo qui s'intéressent au premier conflit mondial sont assez peu nombreux. D'après le recensement effectué par le site Historiagames.com ${ }^{17}$, seulement 59 jeux mettent en scène le premier conflit mondial (Figure 1). C'est peu comparé aux quelque 880 jeux s'intéressant à la Seconde Guerre mondiale. Depuis le début des années 1990, la simulation a été le genre vidéoludique le plus fidèle à la Première Guerre mondiale, là où les années 2000 ont vu le genre de la stratégie s'imposer. Il faut attendre les années 2010 pour voir le genre du jeu d'action s'emparer du conflit, tandis que les jeux d'aventure et de rôle n'ont pas véritablement investi cette période historique.

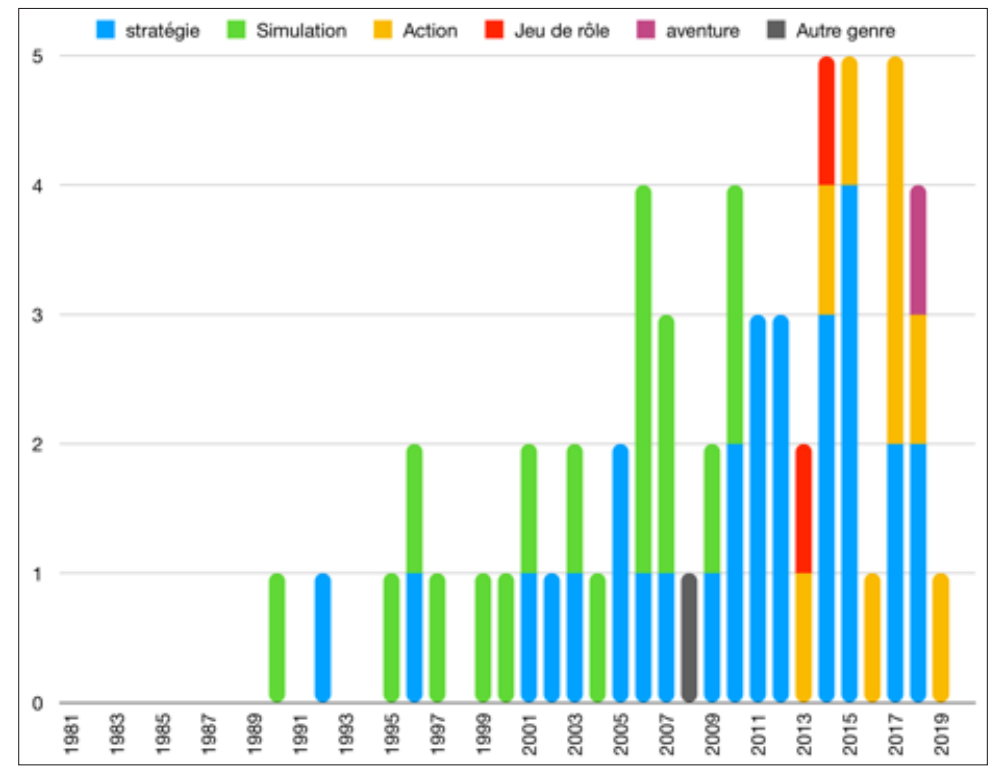

Figure 1 : Chronologie des jeux vidéo traitant de la Première Guerre mondiale par genre

16 Carl E. Schorske, Vienne fin du siècle. Politique et culture, Paris, Le Seuil, 1983, p. 13.

17 Voir la liste sur le site d'Historiagames consacré aux jeux vidéo historique : www.histogames. com. Cette liste mériterait d'être affinée. Je n'ai pas trié les jeux en fonction de leur réussite commerciale, ni écarté les DLC (contenus téléchargeables après la sortie initiale du jeu). 
Si les studios DICE et EA ont fait le choix de la Première Guerre mondiale, leur concurrent direct, Activision-Blizzard, a quant à lui préféré leur opposer Call of Duty: WW2, rebut de leur premier jeu centré sur la Seconde Guerre mondiale. La guerre se trouve de fait dans les enjeux commerciaux. Il est intéressant de mettre en parallèle des ouvres culturelles sorties au même moment, par exemple le film d'Albert Dupontel, Au Revoir Là-Haut, la bande dessinée Souvenirs de Guerre de Jacques Bertran (éditions Flandonnière), ou le jeu vidéo d'Ubisoft, Soldats Inconnus. Mémoires de la Grande Guerre, toutes ces œuvres ne présentant pas la guerre de la même manière ni sous le même angle.

Ainsi, le jeu devient un objet d'histoire; on s'attache à analyser ce qu'il nous apprend de la Première Guerre mondiale, mais aussi et surtout sur la représentation que les créateurs du jeu (scénaristes, level designer, sound designer, game designer) ont de ce conflit. Autrement dit et pour reprendre les travaux de Laury Nuria-André sur l'Antiquité dans les jeux vidéo ${ }^{18}$, il s'agit moins d'étudier la Première Guerre mondiale que sa représentation contemporaine. Partant de cela, il serait intéressant d'interroger avec les élèves la méthode de travail des développeurs. Où se sont-ils informés ? Comment ont-ils réfléchi à la transcription numérique d'un fait historique ? Se sont-ils intéressés aux débats historiographiques ? Beaucoup de questions qui nous feraient déborder le temps imparti relatif au traitement de la Première Guerre mondiale en classe de Troisième... Cinq heures, évaluation comprise.

C'est cette dimension culturelle du jeu qui m'a fait expérimenter une utilisation vidéoludique de la Mémoire de la Première Guerre mondiale. Dans le cadre du Centenaire, les élèves sont allés voir le film Joyeux Noël. Il me paraissait dès lors intéressant de comparer les œuvres afin de montrer des points communs dans la narration et des différences dans les intentions, le film de Carion étant écrit pour montrer la paix, tandis que le jeu d'EA rend hommage aux combattants.

\section{La question de la violence}

Lorsque l'on touche à un jeu de guerre, la question de la violence est légitime. BF1 est un jeu classé PEGI 18, c'est-à-dire que sa vente est déconseillée aux enfants de moins de 18 ans. On peut de fait s'interroger sur la nécessité de mettre des enfants de 14-15 ans devant un tel jeu, d'autant plus que le jeu vidéo est une industrie souvent jugée responsable d'actes de violence comme les tueries aux États-Unis. D’aucuns accusent même les jeux vidéo d'être à l'origine des violences interpersonnelles entre certains de nos élèves. ${ }^{19}$

18 Laury Nuria-André, Game of Rome. Ou l'Antiquité vidéoludique, Caen, Passage(s), 2016.

19 Alexandra Edip, «La violence dans les jeux vidéo rend-elle agressif? ", capital.fr, 29 juin 2017 : https://www.capital.fr/polemik/la-violence-dans-les-jeux-videos-rend-elle-agressif-1234881 (dernière visite le 28/11/2018). 
La première des précautions est de placer les élèves en position d'observateur $\mathrm{du}$ jeu. Ils ne sont pas en situation de donner la mort par leurs actions. La seconde se situe dans l'explication réalisée au préalable et destinée aux enfants et à leurs parents, de la démarche dans laquelle s'inscrit cette séquence. Il y a un effort de pédagogie à faire autour de l'utilisation des jeux de guerre en classe. Dans son article, Stéphanie Trouillard insiste par exemple sur la violence qu'a été pour elle de voir les noms de vrais soldats apparaitre dans le jeu ${ }^{20}$. Certains élèves ont également demandé s'il s'agissait de vrais noms, ce qui nous pousse à croire que le réalisme serait à tel point violent qu'il pourrait être dérangeant. Plus important encore, le jeu vidéo, en ce qu'il met le joueur en situation d'acteur d'une histoire reconstituée, nous invite à remettre en question la violence transmise par les images, les sons et les textes. De plus en plus de jeunes jouent à ces jeux ; les inviter à nourrir une réflexion sur ces objets doit permettre de les inciter à prendre du recul vis-à-vis de ces images. Il est donc nécessaire de faire prendre conscience au jeune public que les jeux vidéo sont des constructions et que la violence qu'ils transportent est également une construction. Une analyse technique du fonctionnement des armes dans le jeu et dans la réalité pourrait permettre cela. L'on peut aussi discuter avec eux des différences de traitement de plusieurs jeux de guerre par le système PEGI. Si BF1 et la série des Call of Duty sont classés PEGI 18, comment se fait-il que Fortnite $^{21}$ soit classé PEGI 12 ? Toutes les violences ne semblent de fait pas se valoir. Il serait toutefois malvenu d'oublier que la Première Guerre mondiale a été une guerre violente et traumatisante ${ }^{22}$. En 2000, Stéphane AudoinRouzeau reprochait aux historiens de la Première Guerre mondiale une trop grande pudeur, en ce qu'ils ne parlaient de la violence et des morts qu'à travers des tableaux et des chiffres. Il souhaitait une histoire plus incarnée :

" Peut-être parce qu'ils portent sur des ordres de grandeur trop importants, peut-être parce qu'ils ont été souvent cités, peut-être enfin parce qu'ils se heurtent à nos puissants réflexes de déréalisation dès lors que nous sommes confrontés aux effets de la guerre, de tels chiffres ne possèdent, curieusement, qu'une capacité d'évocation assez faible. ${ }^{23}$

Relisons à ce sujet Blaise Cendrars :

«Et voilà qu'aujourd'hui j’ai le couteau à la main. [...] J'ai bravé la torpille, le canon, les mines, le feu, les gaz, les mitrailleuses, toute la machinerie anonyme, démoniaque, systématique, aveugle. Je vais broyer l'homme. Mon semblable. Un singe. CEil pour œil, dent pour dent. À nous deux maintenant. À coup

20 Stéphanie Trouillard, «Battlefield 1 n'est pas un jeu sur la Première Guerre mondiale », op. cit.

21 Jeu développé par Epic Games (2017) dans lequel les joueurs combattent d'autres joueurs au sein d'une arène (en solo ou en petit groupe) afin d'être le dernier survivant.

22 Le nombre de personnes portant les séquelles de la Première Guerre mondiale (militaires et civiles) s'élève à plus de 40 millions, 20 millions de morts et 21 millions de blessés.

23 Stéphane Audoin-Rouzeau et Annette Becker, 14-18, retrouver la Guerre, Paris, Gallimard, 2000 , p. 39. 
de poing, à coup de couteau. Sans merci. Je saute sur mon antagoniste. Je lui porte un coup terrible. La tête est presque décollée. J'ai tué un Boche. J'étais plus vif et plus rapide que lui. Plus direct. J'ai frappé le premier. J'ai le sens de la réalité, moi, pote. J'ai agi. J'ai tué. Comme celui qui veut vivre. $»^{24}$

La violence présente dans ce texte est-elle compréhensible par un élève de Troisième ? BF1, malgré ces imperfections, ne permet-il pas au contraire de faire vivre une expérience qui n'est plus possible, heureusement, mais qui peut par la suite être discutée ?

\section{Les enjeux mémoriels}

Ce travail sur BF1 s'inscrit dans le devoir de mémoire. En 2018, nous avons fêté la fin du Centenaire de la Première Guerre mondiale. Le devoir de mémoire a créé une véritable émulation autour du conflit et de son souvenir et a engendré de nombreuses productions culturelles. Sorti en 2016, Battlefield 1 s'insère dans ce processus mémoriel : pourquoi la société DICE et EA ont-elles décidé de développer un jeu sur la Première Guerre mondiale, alors qu'elles n'ont jamais abordé ce conflit auparavant ? La coïncidence est troublante. Ce questionnement permet aussi d'aller plus loin et de poser la question de la déontologie : est-ce qu'il est acceptable de jouer avec la mort des soldats? Si Stéphanie Trouillard fut choquée par les noms de vrais soldats présents dans la première mission du jeu, n'est-ce pas aussi une manière de rappeler la mémoire des soldats tombés au combat, comme le font les monuments aux morts?

Pour aller plus loin, Battlefield 1 permet une approche du concept historique de mémoire. Chaque " récit de guerre " est raconté par un vétéran. Durant la première mission, un Harlem Hellfghters se réveille à l'hôpital et se souvient des violences qu'il a vues. Dans "De l'acier et de sang ", Daniels, un chauffeur de taxi se souvient des événements traumatisants qu'il a vécus en voyant ces gants. Dans la mission "Avanti Savoia" qui se déroule dans les Alpes, un père de famille raconte à sa fille comment lui et son frère ont pris d'assaut une forteresse dans les Alpes. Tout au long de la mission, alors que l'on revit les événements vécus par le père, il nous raconte ses faits et gestes. Cette mise en scène du souvenir et de la mémoire est très intéressante à analyser avec les élèves ; elle permet d'appréhender la réalité des traumatismes et des traumatisés de guerre. Mais plus encore, l'on peut imaginer que le joueur n'interprète pas ce que ces vétérans ont vécu, mais plutôt ce dont ils se souviennent. La mémoire est quelque chose d'imparfait, elle est par définition incomplète. Ainsi, il y a toujours une place pour l'imagination : tous les défauts du jeu, les aspects irréalistes peuvent alors être "pardonnés ». La mémoire n'est pas l'histoire : si cela peut sembler exagéré, c'est à mon avis une piste de discussion qu'il ne faut pas négliger ${ }^{25}$.

24 Blaise Cendrars, J'ai tué, Paris, G. Crès, 1918, p. 21.

25 Ce travail sur la place de la mémoire dans les récits de guerre peut permettre des parallèles avec 


\section{Conclusion}

Ce travail, perfectible, a permis une première approche scolaire du travail critique des jeux vidéo. Utilisé dans un premier temps comme un stimulant, le jeu suscite la curiosité des élèves, y compris décrocheurs. Il permet aussi de créer une rupture avec les cours précédents. C'est un média qui ne dit pas son nom : c'est un jeu. Il est dès lors intéressant de faire prendre conscience aux élèves que le jeu produit un discours sur le passé. Cette séquence permet autant un travail historique sur la Première Guerre mondiale que sur la mémoire et la représentation vidéoludique de la Grande Guerre. Validée par l'Inspection académique, celle-ci s'inscrit en effet dans le parcours d'Éducation aux Médias et à l'Information (EMI). Comme le relève Laurent Turcot dans son ouvrage critique d'Assassin's Creed Unity pour lequel il a officié comme consultant : " [les jeux vidéo sont] une porte d'entrée ouverte sur le passé. Gageons que certains joueurs auront envie d'en savoir plus, recueilleront quelques informations sur le web, d'autres dans des livres, et à partir de là dans d'autres livres encore. » ${ }^{26}$

Le jeu vidéo est un objet d'histoire, mais peut aussi être un outil pédagogique. Nous aurions tort de ne pas l'utiliser, d'autant plus lorsqu'on sait qu’il n’a pas été conçu pour cela.

William Brou

Professeur d'Histoire-Géographie

Collège Antoine Audembron (63)

william.brou@gmail.com

William Brou est diplômé d'un master 2 Recherche en Histoire médiévale (2012) et titulaire du CAPES. Il enseigne en collège-lycée. Actuellement au collège Antoine Audembron (Thiers 63), dans l'académie de Clermont-Ferrand, il utilise les jeux vidéo dans son enseignement de l'histoire. Il crée des scénarios pédagogiques utilisant des jeux vidéo, surtout grand public, dans le but d'appréhender les savoirs disciplinaires, éduquer à l'esprit critique et faire de l'enseignement aux médias et à l'information. En parallèle, il anime une chaîne YouTube : Histoire en Jeux (https://www.youtube.com/c/HistoireenJeux) depuis janvier 2017. Sur cette chaîne, il analyse et critique des jeux vidéo qui utilisent l'histoire. Membre des Clionautes, il anime pour eux, une veille scientifique sur les réseaux sociaux autour de la ludification de l'enseignement de l'histoire.

Adresse électronique : william.brou@ac-clermont.fr

le film, Les Fragments d'Antonin de Gabriel le Bomin (2006), dans lequel on suit le parcours d'un traumatisé de guerre qui revit des épisodes traumatiques.

26 Jean-Clément Martin et Laurent Turcot, Au cour de la Révolution. Les leçons d'histoire d'un jeu vidéo, Paris, Vendémiaire, 2015, p. 124. 


\section{Résumé}

Les programmes de collège et lycée nous invitent à étudier l'expérience combattante durant la Première Guerre mondiale. Ils demandent aussi à éveiller l'esprit critique et à faire découvrir les langages artistiques à nos élèves. Battlefield 1 est un jeu de tir à la première personne qui met le joueur dans la peau de plusieurs soldats de la Première Guerre mondiale. Utiliser Battlefield 1 en classe permet dans un premier temps de faire réaliser aux élèves un "fact-checking " avec des documents d'archives. La reconstitution vidéoludique de la Première Guerre mondiale permet d'aborder la construction du jeu en analysant le game design, le level design et le sound design. Le jeu est finalement une porte d'entrée vers des questionnements autour la mémoire du conflit.

Mots-clés

Première Guerre mondiale, Jeux vidéo, Battlefield 1, Esprit critique, Fact-checking.

\section{Abstract}

Middle and high school programs invite us to study the fighting experience during the First World War. They also ask to awaken the critical mind and to discover the artistic languages for our students. Battlefield 1 is a first-person shooter video game which puts the player in the role of several soldiers of the First World War. Using Battlefield 1 in classroom allows students to perform factchecking with archival documents. Because of the videoludic reconstitution of the First World War, videogames allow to approach the construction of the game by analyzing game design, level design and sound design. Finally, this video game is a way to question the memory of this conflict.

\section{Keywords}

First World War, Video Games, Battlefield 1, Critical Mind, Fact-checking. 